\title{
Etude du déferlement par modélisation numérique 2D et 3D
}

Pierre Lubin ${ }^{1}$, Stéphane Abadie ${ }^{2}$, Stéphane Vincent ${ }^{3}$, Jean-Paul Caltagirone ${ }^{4}$

${ }^{1}$ Doctorant, ${ }^{3}$ Maitre de conférences, ${ }^{4}$ Professeur, Ecole Nationale Supérieure de Chimie et de Physique de Bordeaux, Laboratoire de Modélisation Avancée des Systèmes Thermiques et des Écoulements Réels (MASTER), 16 avenue PeyBerland, 33607 PESSAC CEDEX.

Tél. : (+33)-5-56-84-66-67

E-mail :lubin@lmaster.u-bordeaux.fr

${ }^{2}$ Maitre de conférences, ISA-BTP, Laboratoire des Sciences Appliquées au Génie Civil (LASAGEC), Parc de Montaury, 64600 ANGLET.

Tél. : (+33)-5-59-57-70-48

E-mail : stephane.abadie@univ-pau.fr

\section{Résumé}

Cet article expose les résultats obtenus sur l'étude du déferlement 2D et les premières simulations en $3 \mathrm{D}$. Les équations de Navier-Stokes sont résolues à la fois dans l'air et dans l'eau en utilisant la méthode du Lagrangien Augmenté, le suivi de l'interface étant réalisé par la méthode VOF-CIAM (Volume Of Fluid Calcul d'Interface Affine par Morceaux) qui permet de gérer la reconnexion de l'interface. Le déferlement $2 \mathrm{D}$, puis $3 \mathrm{D}$, par initialisation d'une onde sinusoïdale instable, est alors étudié.

\begin{abstract}
This paper shows the results obtained for simulating breaking of $2 \mathrm{D}$, and then 3D, waves. The Navier-Stokes equations are solved in air and water, the interface tracking being achieved by the VOF-PLIC method (Volume Of Fluid - Piecewise Linear Interface Calculation), which is able to handle with interface reconnections. A $2 \mathrm{D}$, then $3 \mathrm{D}$, study of breaking waves is presented, using initial conditions corresponding to unstable sinusoidal waves of large amplitudes.
\end{abstract}

\section{Introduction}

Le déferlement des vagues est un phénomène fascinant qui joue un rôle de première importance dans les processus côtiers. Il est à l'origine de courants très intenses, responsables de la majeur partie du transport sédimentaire dans cette zone. Les ouvrages côtiers sont en outre régulièrement soumis à des impacts infligés par les brisants et le déferlement des vagues. Or, bien que largement étudié depuis une vingtaine d'années, le déferlement est un phénomène encore très difficile à appréhender, aussi bien numériquement qu'expérimentalement.

Différentes études ont permis d'obtenir des résultats appréciables avant l'impact ainsi que quelques mètres après l'impact, dans la zone de surf dite 
« interne » (voir Peregrine (1983) et Battjes (1988) pour une synthèse de ces travaux). Or la majeure partie de la dissipation de la vague a lieu dans la zone dite de transition, c'est à dire immédiatement après l'impact.

Du point de vue numérique, on peut aborder cette zone de transition à l'aide de modèles reposant sur les équations de Navier-Stokes et un suivi d'interface autorisant les reconnexions (voir Abadie et al., 1998, Lin \& Liu, 1998, Chen et al., 1999, Christensen \& Deigaard, 2000, Guignard et al., 2001).

L'étude présentée dans cet article participe à l'effort de recherche pour décrire cet écoulement complexe. La méthodologie employée repose sur une simulation numérique «directe» des équations de Navier-Stokes diphasiques bidimensionnelles et un suivi d'interface volumique. Après avoir rapidement introduit les fondements de cette modélisation, des résultats concernant l'écoulement généré par déferlement sont analysés.

\section{Présentation de la méthode}

\subsection{Résolution de l'écoulement}

Le problème considéré est l'écoulement de deux fluides incompressibles non miscibles. Le système d'équations régissant l'écoulement diphasique dans chacune des phases sont les équations de Navier-Stokes, accompagnées de la condition d'incompressibilité :

$$
\left\{\begin{array}{l}
\nabla \cdot \mathbf{U}=0 \\
\rho\left(\frac{\partial \mathbf{U}}{\partial t}+(\mathbf{U} \cdot \nabla) \mathbf{U}\right)=\rho \mathbf{g}-\nabla p+\nabla \cdot \mu\left(\nabla \mathbf{U}+\nabla^{T} \mathbf{U}\right)+\sigma \kappa \delta_{i} n_{i}
\end{array}\right.
$$

où $\rho$ est la densité et $\mu$ la viscosité dynamique, $U$ le champ de vitesse et $p$ le champ de pression. $\sigma$ est la tension de surface, constante sur l'interface liquidegaz. Il est à noter que la viscosité et la densité sont constantes dans les deux phases de l'écoulement. Ce sont des fonctions du point que l'on détermine à partir de la position de l'interface entre les deux phases. Dans cette étude, les équations de Navier-Stokes sont résolues soit par la méthode du Lagrangien Augmenté, soit par un algorithme de projection (Chorin, 1968).

\subsection{Suivi de l'interface}

D'un point de vue interface, le déferlement se caractérise par des déconnections et reconnections créant de nouvelles surfaces de toutes formes et de toutes tailles. Parmi les différentes méthodologies employées pour décrire l'évolution temporelle de l'interface eau / air, nous utilisons des méthodes de type VOF (Volume Of Fluid) (Hirt \& Nichols, 1981). L'interface y est décrite par une fonction $\mathrm{F}$ traduisant le coefficient de remplissage de l'eau dans chaque maille d'un maillage fixe. Cette représentation permet de traiter naturellement le problème de reconnections de l'interface sur elle-même en comparaison des méthodes à traceurs surfaciques. On définit ainsi la fonction binaire $\mathrm{F}$ telle que 
$F=1$, si le point est dans l'eau, et $F=0$, si le point est dans l'air.

La valeur moyenne de la fonction $F$ sur un volume représente alors la fraction volumique d'eau dans ce volume. L'interface étant une surface matérielle et la fonction $\mathrm{F}$ une fonction caractéristique de cette interface, l'équation d'évolution temporelle de F s'écrit :

$$
\frac{\partial F}{\partial t}+U \cdot \nabla F=0
$$

Afin de pallier aux défauts tel que la diffusion numérique, intrinsèque aux schémas utilisés pour discrétiser l'équation (3), l'algorithme VOF-CIAM $(\mathrm{Li}$, 1995) a été programmé. Le transport Lagrangien utilisé dans cette méthode permet d'obtenir une précision supérieure aux méthodes VOF antérieures utilisant un calcul de flux pour l'étape d'advection.

\section{Modélisation du déferlement}

\subsection{Conditions aux limites}

Le déferlement est étudié dans un domaine bidimensionnel semi-périodique (dans la direction horizontale). Une condition de symétrie est imposée sur le fond et une condition libre à la frontière supérieure de notre domaine. La hauteur du domaine de calcul est ajustée afin d'éviter un effet de confinement « artificiel» .

Nous utilisons un critère de CFL (Courant-Friedrichs-Levy) de $0,3:$ cette condition, qui correspond au rapport de la taille de la maille par la vitesse, impose un déplacement de l'interface inférieur à 0,3 fois la maille par pas de temps de calcul. Le pas de temps est donc recalculé à chaque itération afin de satisfaire cette condition nous assurant une bonne stabilité de la méthode VOF-CIAM.

\subsection{Conditions initiales}

L'onde incidente est obtenue à partir d'une solution initiale sinusoïdale à forte cambrure (Cockelet, 1979, Vinje \& Brevig, 1981, Abadie et al., 1998, Chen et al., 1999, Abadie, 2001). Toutes les quantités caractérisant l'onde initiale sont donc calculées sur la base de la théorie linéaire. Une telle solution est instable, car le champs de vitesses initialisé dans l'eau n'est pas en équilibre avec le profil de surface libre imposé.

Les variables de référence de notre problème sont la célérité c de l'onde incidente initiale, sa période $T$ et sa longueur $L$, les masses volumiques de l'air et de l'eau ( $\rho_{\text {air }}$ et $\rho_{\text {eau }}$ ), les viscosités dynamiques de l'air et de l'eau ( $\mu_{\text {air }}$ et $\mu_{\text {eau }}$ ). d désigne la profondeur et $\mathrm{H}$ la hauteur de crête à creux.

L'écoulement est donc caractérisé par les nombres adimensionnels regroupés dans le tableau 1. Les deux derniers nombres sont, respectivement, 
représentatifs du degré de non-linéarité de l'onde et de l'influence du fond sur le mouvement ondulatoire.

\begin{tabular}{|c|c|c|c|c|c|}
\hline Reynolds & Froude & $\begin{array}{c}\text { Rapport de } \\
\text { densité }\end{array}$ & $\begin{array}{c}\text { Rapport de } \\
\text { viscosité }\end{array}$ & $\begin{array}{c}\text { Cambrure } \\
\text { initiale }\end{array}$ & $\begin{array}{c}\text { Effet de la } \\
\text { profondeur }\end{array}$ \\
\hline $\operatorname{Re}=\frac{\rho c L}{\mu_{\text {eau }}}$ & $F r=\frac{c}{\sqrt{g L}}$ & $\frac{\rho_{\text {air }}}{\rho_{\text {eau }}}$ & $\frac{\mu_{\text {air }}}{\mu_{\text {cau }}}$ & $\frac{H}{L}$ & $\frac{d}{L}$ \\
\hline
\end{tabular}

Tableau 1 : nombres adimensionnels de la simulation

Table 1 : simulation characteristic numbers

Plusieurs données de notre problème sont fixes : les rapports

$\frac{\mu_{\text {air }}}{\mu_{\text {eau }}}=1,85 \cdot 10^{-2}$ et $\frac{\rho_{\text {air }}}{\rho_{\text {eaur }}}=1,1768 \cdot 10^{-3}$.

En revanche, les nombres de Reynolds réels sont encore hors de portée des simulations à la fois d'un point de vue physique (nombre de mailles insuffisant, modélisations de la turbulence inadaptée) mais également semble t-il d'un point de vue numérique.

Nous sommes donc limités en dimensions car le nombre de Reynolds dépend directement de la longueur de l'onde incidente initiale. Ceci n'est pas forcément rédhibitoire puisque, comme l'a montré Stive (1985) par ses travaux expérimentaux, les effets d'échelle sont faibles pour des vagues de dimensions comprises entre 0,1 et 1,5 mètres d'amplitude.

\subsection{Présentation des résultats}

\subsubsection{Résultats 2D}

La validation de l'ensemble de la méthode a déjà fait l'objet d'une publication (Abadie et al., 1998). Une étude de propagation d'ondes permanentes non linéaires et une description précise d'un ensemble de phénomènes, tel que le jet secondaire ou « splash-up », a été réalisée.

Actuellement, une des difficultés principales semble donc être une limite numérique en nombre de Reynolds. En effet, pour des nombres de Reynolds supérieurs ou égaux à $10^{6}$ les méthodes de résolution utilisées (Lagrangien Augmenté et Projection) ne parviennent pas à résoudre correctement l'écoulement au voisinage de l'interface lorsque celle ci est soumise à de fort cisaillement (phase d'éjection).

Abadie et al. (1998) a montré qu'en faisant usage d'une viscosité artificielle introduite dans les deux phases, tout en conservant le rapport des viscosités, ces instabilités pouvaient être amorties sans pour autant modifier les 
caractéristiques principales de l'écoulement. En adoptant la même démarche, nous proposons, dans cet article, des résultats obtenus pour des Reynolds de $10^{4}$.

En jouant sur le paramètre cambrure initiale, il est possible de générer plusieurs types de déferlement, allant du déferlement fortement plongeant au déferlement déversant. Nous avons fixé le nombre $\mathrm{d} / \mathrm{L}$ à 0.17 (permettant d'obtenir un déferlement correspondant approximativement à ce qui peut être observé sur une plage (i.e. $\mathrm{H} / \mathrm{d}=0.8$ )) et nous avons fait varier la cambrure initiale $\mathrm{H} / \mathrm{L}$ afin d'obtenir trois types de déferlement bien distinct.

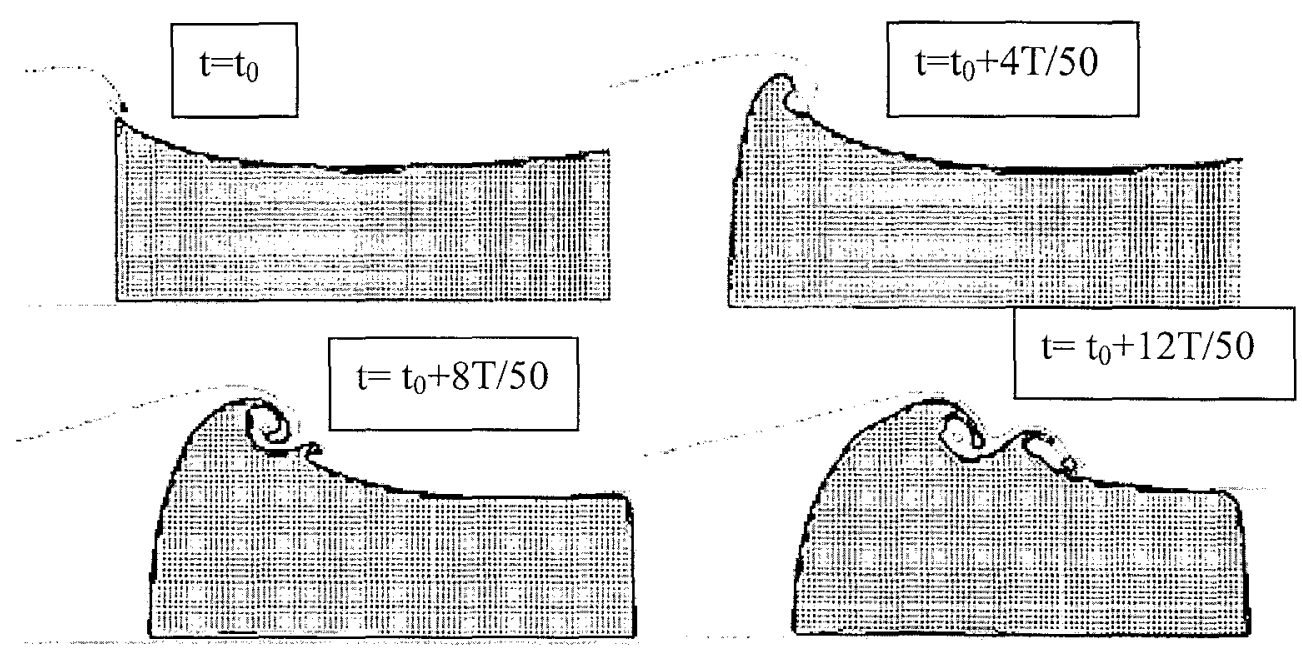

Figure 1 : Mélange des masses d'eau dans le déferlement faiblement plongeant

Figure 1 : mass mixing in a plunging wave

$\mathrm{Re}=10^{4}, \mathrm{H} / \mathrm{L}=0.10, \mathrm{~d} / \mathrm{L}=0.17,200 \times 100, \sigma=0$, projection, $\mathrm{t}_{0}=0.56 \mathrm{~T}$

Les figures 1 à 3 présentent les résultats concernant l'étude du jet secondaire par suivi d'un traceur numérique. Dans Abadie et al. (1998), le jet secondaire est identifié comme provenant exclusivement du creux de la vague et non du jet. Mais le déferlement étudié est violent et intervient dans très peu d'eau. Dans cet article, l'éclaboussure apparaît dans les trois cas constituée d'un mélange du jet initial et de la partie basse de la vague. Il paraît donc probable que ce cas de figure soit le plus fréquent dans le déferlement réel. Un autre point intéressant à relever est la faible profondeur de pénétration du jet dans tous les type de déferlement y compris fortement plongeant (Abadie et al. 1998). En fait, les vitesses importantes qui sont générées sur le fond dans la plupart des déferlement apparaissent essentiellement liées à la descente du tourbillon horizontal généré par la reconnexion plutôt qu'à la pénétration du jet initial. 


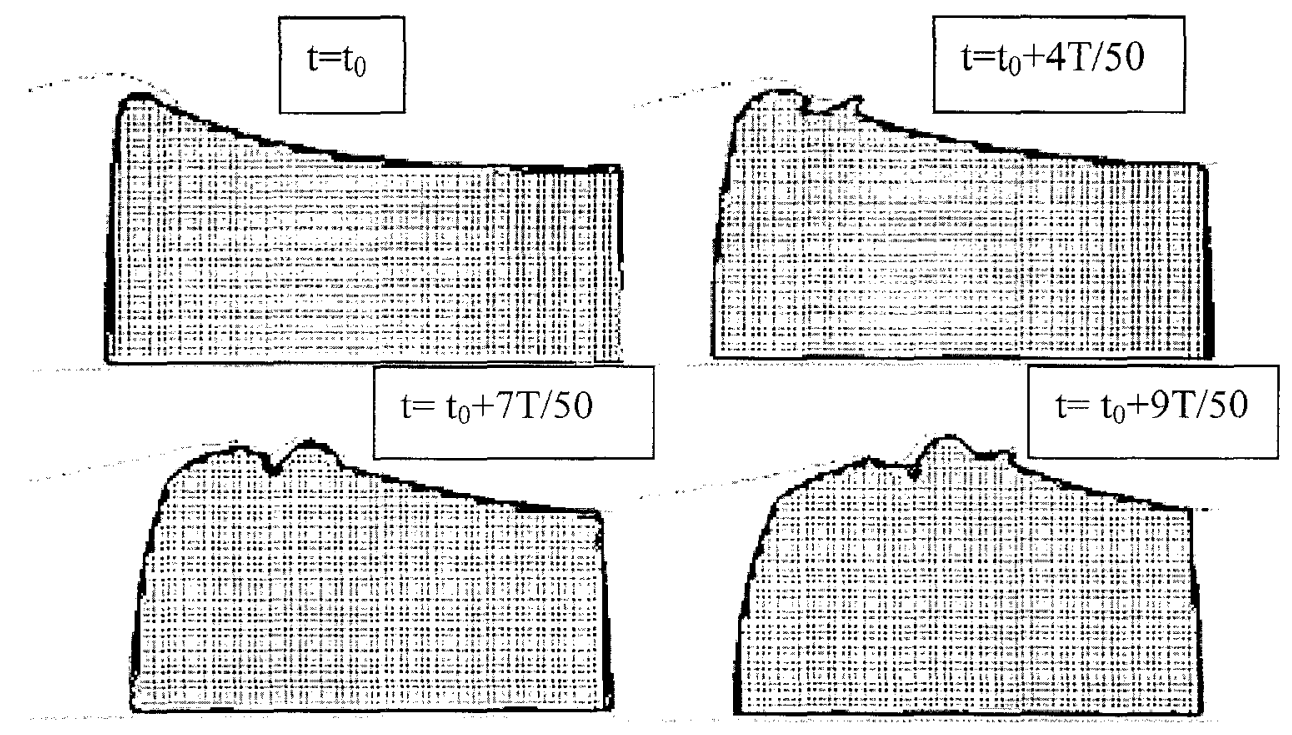

Figure 2 : Mélange des masses d'eau dans le déferlement déversant Figure 2 : mass mixing in a spilling breaker

$\mathrm{Re}=10^{4}, \mathrm{H} / \mathrm{L}=0.06, \mathrm{~d} / \mathrm{L}=0.17,200 \times 100, \sigma=0$, projection, $\mathrm{t}_{0}=\mathrm{T}$

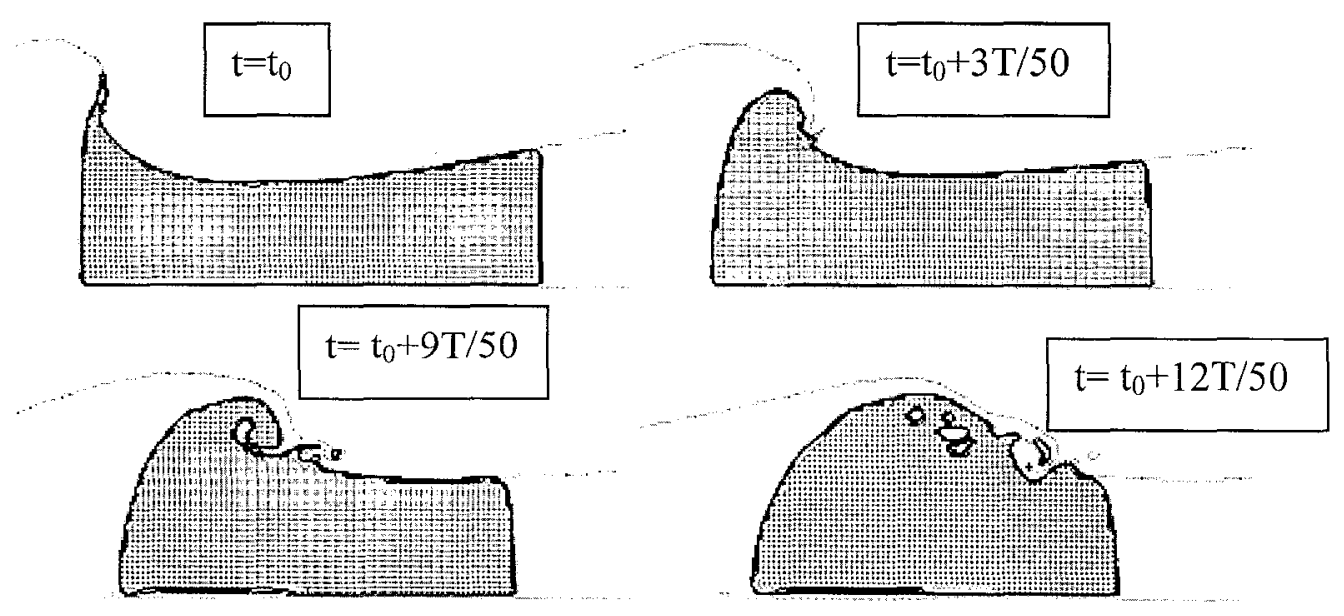

Figure 3 : Mélange des masses d'eau dans le déferlement gonflant Figure 3 : mass mixing in a surging breaker

$\operatorname{Re}=10^{4}, \mathrm{H} / \mathrm{L}=0.14, \mathrm{~d} / \mathrm{L}=0.17,200 \times 100, \sigma=0$, projection, $\mathrm{t}_{0}=0.38 \mathrm{~T}$

\subsubsection{Résultats 3D}

Nous ne présentons ici que les toutes premières visualisations d'un calcul 3D sur un maillage $400 \times 3 \times 200$. Le nombre de Reynolds est approximativement $10^{5}$ et les nombres $\mathrm{d} / \mathrm{L}$ et $\mathrm{H} / \mathrm{L}$ sont égaux à 0,13 et la tension superficielle $\sigma=7,5.10^{-2}$ $\mathrm{N} . \mathrm{m}^{-1}$. Le nombre de points semble cependant inférieur à ce qu'il faudrait, puisque l'interface devient instable au moment du déferlement. Nous procédons à 
l'initialisation de la même onde instable qu'en 2D, en projetant sur l'axe transversal. Cet exemple illustre bien la limite numérique qui semble intervenir pour ce problème aux alentours de $\mathrm{Re}=10^{5}-10^{6}$ lorsque à la fois l'air et l'eau sont simulés. Les travaux ne simulant que le mouvement de l'eau (cf. Christensen \& Deigaard, 2001) ne sont pas gênés par cette difficulté.

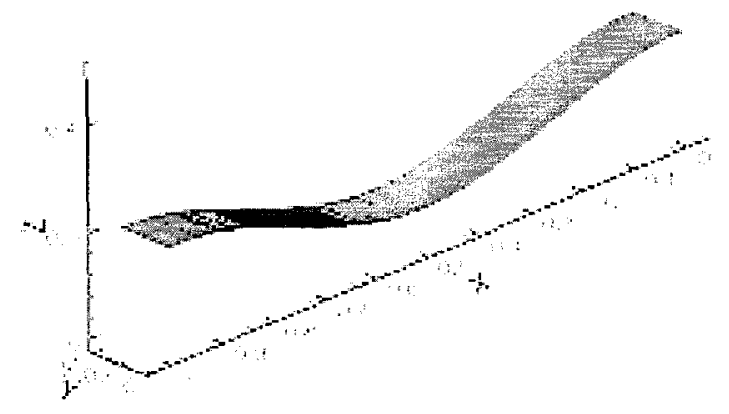

a)

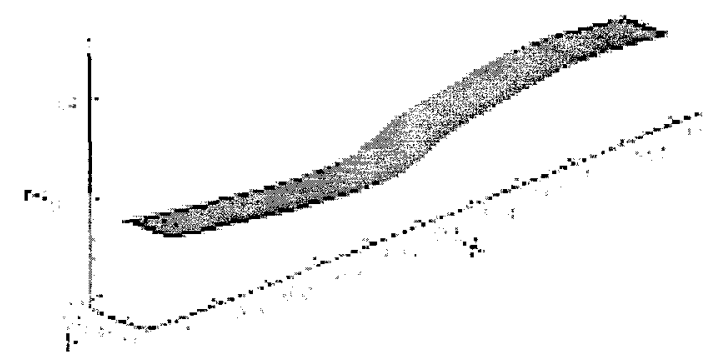

b)

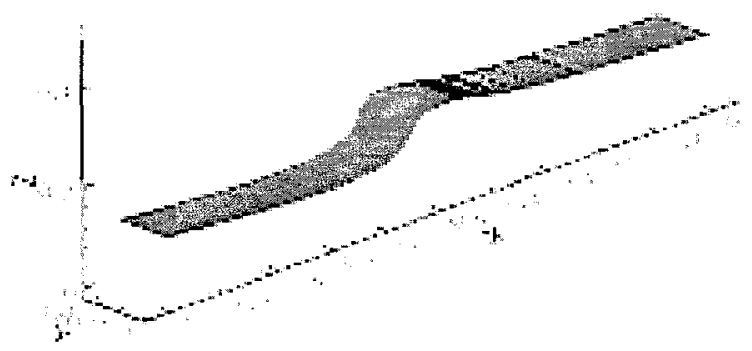

c)

Figure 4 : Simulation 3D

Isosurface $\mathrm{F}=0,5$; a) $\mathrm{t}=0 \mathrm{~s}$; b) $\mathrm{t}=0,2 \mathrm{~s}$; c) $\mathrm{t}=0,3 \mathrm{~s}$

\section{Conclusion et perspectives}

Dans cette communication, le déferlement est modélisée par les équations de Navier Stokes à deux phases et un suivi d'interface de type VOF. Des résultats concernant le jet secondaire pour les trois types de déferlement classique sont présentés. On trouve en particulier que cette éclaboussure est dans tous les cas un mélange du jet initial et de la partie basse de la vague. D'autre part le jet ne pénètre jamais profondément la colonne d'eau et les forte vitesses qui interviennent sur le fond sont dues aux tourbillons créés par la reconnexion. Ces résultats sont encourageants et le travail est amorcé en trois dimensions. 
La suite a apporter à notre étude sera d'effectuer une étude précise de tous les types de déferlement en faisant varier les nombres d/L et H/L. Nous observerons les temps de déclenchement, les taux d'énergie dissipée ainsi que la turbulence générée. Pour ce faire, nous développerons des modèles de SGE (Simulation des grandes Echelles, ou LES en anglais). Ceux-ci seront couplés avec une méthode de raffinement local développée au laboratoire (Vincent, 2000) qui nous permettra de pallier aux problèmes d'irrégularités de notre interface. Une étude comparative sera menée avec d'autres méthodes de suivi d'interface, telle que TVD (Total Variation Diminishing) disponible dans notre code (Vincent, 1999).

\section{Références bibliographiques}

Abadie S. , 2001, "Numerical modelling of the flow generated by plunging breakers ", Proceedings Coastal Dynamics 2001, p202-211, Lund, Suède.

Abadie S., Caltagirone J. P. et Watremez P., 1998, Mécanisme de génération du jet secondaire ascendant dans un déferlement plongeant, C. R. Acad. Sci. Paris, t. 326, Série II b, p. 553-559.

Battjes, J. A., 1988, Surf-zone dynamics, Ann. Rev. Fluid Mech., 20, 257-293.

Chen, G. et al., 1999, Two-dimensional Navier-Stokes simulation of breaking waves, Physics of Fluids, 11, 121-133.

Christensen, E. D., Deigaard, R., 2001, Large eddy simulation of breaking waves, Coastal Eng., 42, 53-86.

Cokelet, E. D., 1979, Breaking waves, the plunging jet and interior folw-field, Mechanics of Wave-Induced Forces on Cylinders, Ed. T. L. Shaw, 287-301.

Galvin, C. J., 1968, Breaker type classification on three laboratory beaches, J. of Geophysical Research, 73, 3651-3659.

Guignard, S. et al., 2001, Solitary wave breaking on sloping beaches : 2-D two phase flow numerical simulation by SL-VOF method, Eur. J. Mech. B-Fluids, 20, 57-74.

Hirt, C. W., Nichols, B. D., 1981, Volume of fluid method for the dynamic of free boundaries, J. Comp. Phys., 39, 323-345.

Lin, P., Liu, P. L.-F., 1998, A numerical study of breaking waves in the surf zone, $J$. Fluid Mech., 359, 239-264.

New, A. L. et al., 1985, Computations of overturning waves, J. Fluid Mech., 150, 233 251.

Peregrine, D. H., 1983, Breaking waves on beaches, Ann. Rev. Fluid Mech., 15, 149178.

Vincent, S., Caltagirone, J.-P., 1999, Efficient solving method for unsteady incompressible interfacial flow problems, Inter. J. for Numer. Meth. Fluids, 30, 795-811.

Vincent, S., Caltagirone, J.-P., 2000, A one cell local multigrid method for solving unsteady incompressible multi-phase flows, J. Comp. Phys., 163, 172-215.

Vinje, T., Brevig, P., 1981, Numerical simulation of breaking waves, J. Adv. Water Resour., 4, 77-82.

Stive, M. J. F., 1985, A scale comparison of waves breaking on a beach, Coastal Eng., 9, 151-158. 